\title{
Prevalência de excesso de peso em pessoas que vivem com HIV: uma revisão sistemática
}

\author{
Prevalence of overweight between people living with HI: \\ a systematic review
}

Fernanda Cristina Gomes Pinhal, ${ }^{1}$ Maria Georgina Marques Tonello, ${ }^{1}$ Cléria Maria Lôbo Bittar, Helena Siqueira Vassimon

Universidade de Franca (Unifran), Franca, SP, Brasil.

Recebido em: 18/08/2016 / Aceito em: 15/09/2016 / Publicado em: 18/10/2016

nandapinha/@hotmail.com

\section{RESUMO}

O uso da terapia antirretroviral (TARV) trouxe meIhorias significativas para a qualidade de vida de pessoas que vivem com HIV, que não mais se caracterizam pela subnutrição. Após 20 anos do uso deste medicamento, estudos vêm descrevendo aumento de excesso de peso nesta população, o que tem despertado o interesse de diversos pesquisadores. Objetivo: identificar prevalência de excesso de peso em pessoas que vivem com HIV. Método: revisão sistemática realizada através da base de dados PubMed e dos descritores prevalence, HIV, nutricional status e obesity. Foram incluídos os artigos cujos estudos foram realizados com adultos que vivem com HIV; que continham as palavras-chave em seu título, resumo ou texto; que fossem artigos completos, originais e publicados nos idiomas, inglês, português e espanhol. Foram pesquisados artigos publicados nos últimos dez anos. A busca foi realizada durante a primeira quinzena de novembro de 2015. Foram excluídos artigos realizados com população infantil, adolescente e gestante, artigos incompletos, de tese, dissertação e de revisão sistemática. Resultados: ao fim da análise de pares, seis artigos foram selecionados, sendo cinco deles realizados no Brasil e apenas um no México. Os estudos apontaram prevalência de excesso de peso em pessoas que vivem com HIV que variou entre $25 \%$ e $48,3 \%$, média de $38,7 \%$ dos participantes das pesquisas. Considerações finais: a prevalência de excesso de peso em pessoas que vivem com HIV assemelha-se à taxa de prevalência da população geral, o que sugere necessidade de atenção especial para garantir a quali- dade de vida desta população.

Palavras-chave: Prevalência; HIV; Estado Nutricional; Obesidade.

\section{ABSTRACT}

The use of antiretroviral therapy (ART) has brought significant improvements to the quality of life of people living with HIV, which is no longer characterized by malnutrition. After 20 years of use of this medicine, studies have described an increase of overweight in this population, which has aroused the interest of many researchers. Objective: identify the prevalence of overweight in people living with HIV. Method: systematic review through PubMed database using the descriptors: prevalence and HIV and nutritional status and obesity. We included papers whose studies were conducted with adults living with HIV, containing the keywords in title, abstract or text. Only papers which were full papers, original and published in English, Portuguese and Spanish language were selected. We search for papers published in the last ten years. The search was conducted during the first half of November 2015. We excluded papers made with child population, adolescents and pregnant women, incomplete papers, thesis, dissertation, systematic review. Results: at the end of pair analysis six papers were selected, five of them performed in Brazil and only one in Mexico. The studies showed prevalence of overweight in people living with HIV ranging between $25 \%$ and $48.3 \%$, average $38.7 \%$ of participants of the research. Closing remarks: the 
prevalence of overweight in people living with HIV is similar to the prevalence rate of the general population, which suggests the need for special attention to ensure the quality of life of this population.

Keywords: Prevalence; HIV; Nutritional Status, Obesity.

\section{INTRODUČ̃̃}

Após vinte anos do uso da terapia antirretroviral (TARV), o HIV (vírus da imunodeficiência humana) não mais se caracteriza pelo excesso de magreza e desnutrição, porém a pessoa que vive com HIV (PV/HIV) vem tendo que lidar com novos desafios do uso da medicação. Entre estes desafios, a síndrome lipodistrófica do HIV, relacionada a dislipidemias, resistência insulínica e alterações de composição corporal têm sido bastante estudadas. ${ }^{1}$

A síndrome lipodistrófica do HIV que define-se pela distribuição irregular de gordura corporal e releva-se, tanto em forma lipoatrófica (perda de tecido adiposo), quanto hipertrófica (acúmulo do tecido adiposo). ${ }^{2}$ As anormalidades morfológicas se manifestam em diferentes regiões do corpo. A lipoatrofia é mais comum na face e membros. Já, a lipohipertrofia se faz presente na região abdominal e dorsocervical, também conhecida como giba. ${ }^{3}$

Além destes aspectos, o excesso de peso também vem acometendo esta população, levando a mudanças no estado nutricional e hábitos alimentares que nunca haviam sido descritos. "Verifica-se que o sobrepeso é o principal desvio do estado nutricional nesta população, atingindo $30,5 \%$ dos pacientes".

Pesquisas relatam possíveis fatores associados ao excesso de peso em PV/HIV, sendo eles: ingestão dietética; fatores fisiológicos, psicológicos, genéticos e condições ambientais e também o desejo de não parecer magro demais. ${ }^{5-7}$ Múltiplos fatores podem estar envolvidos no excesso de peso e mudanças na distribuição de gordura corporal, em usuários de antirretrovirais. Há evidências de que o uso de antirretrovirais esteja diretamente associado à síndrome lipodistrófica, porém não há evidências de associação da medicação com excesso de peso. 7,8

Estudos relatam que PV/HIV tem vivenciado estilo de vida semelhante ao da população geral, o que inclui as taxas de prevalência de obesidade. ${ }^{7}$ Neste contexto, o objetivo do presente estudo foi realizar revisão sistemática sobre a prevalência de excesso de peso em pessoas que vivem com HIV.

\section{MÉTODO}

Desenvolveu-se um estudo de revisão sistemática de literatura. A pergunta que direcionou a revisão foi: "qual a prevalência de excesso de peso em pessoas que vivem com HIV (PV/HIV)?".

\section{Critérios de Inclusão e Exclusão}

Foram pesquisados estudos que avaliaram o estado nutricional e excesso de peso em PV/HIV, através de medidas antropométricas e índice de massa corporal (IMC). Os critérios de inclusão foram: artigos completos originais, publicados nos últimos 10 anos (2005-2015) em idiomas inglês, português ou espanhol. Os artigos deveriam envolver pessoas infectadas pelo HIV, que fossem adultas, de ambos os sexos, utilizando o índice de massa corporal como método para avaliar o estado nutricional. A busca bibliográfica utilizou as seguintes palavras-chave "prevalence and HIV and nutricional status and obesity". As palavras-chave deveriam estar presentes no título, resumo ou texto. A busca foi realizada, durante a primeira quinzena de novembro de 2015, na base de dados PubMed.

\section{Estratégia de busca e seleção dos estudos}

Para a busca dos artigos, foi utilizada a base de dados PubMed e as palavras-chave "prevalence and HIV and nutricional status and obesiy". Os artigos rejeitados na primeira triagem foram os que, a partir da análise do título e do resumo, apresentaram inadequação aos critérios de inclusão ou presença de algum dos critérios de exclusão. Nos casos de incerteza ou discordância, o texto completo do artigo era consultado para confirmar sua elegibilidade. Posteriormente foi realizada análise de pares dos artigos selecionados na primeira triagem.

\section{Tabulação dos Dados}

A estratégia de pesquisa encontrou 29 artigos não duplicados, localizada por meio de consulta feita na base de dados PubMed. Ao final, 06 artigos de estudos originais foram incluídos na revisão. O processo completo de seleção dos estudos está descrito na figura 1.

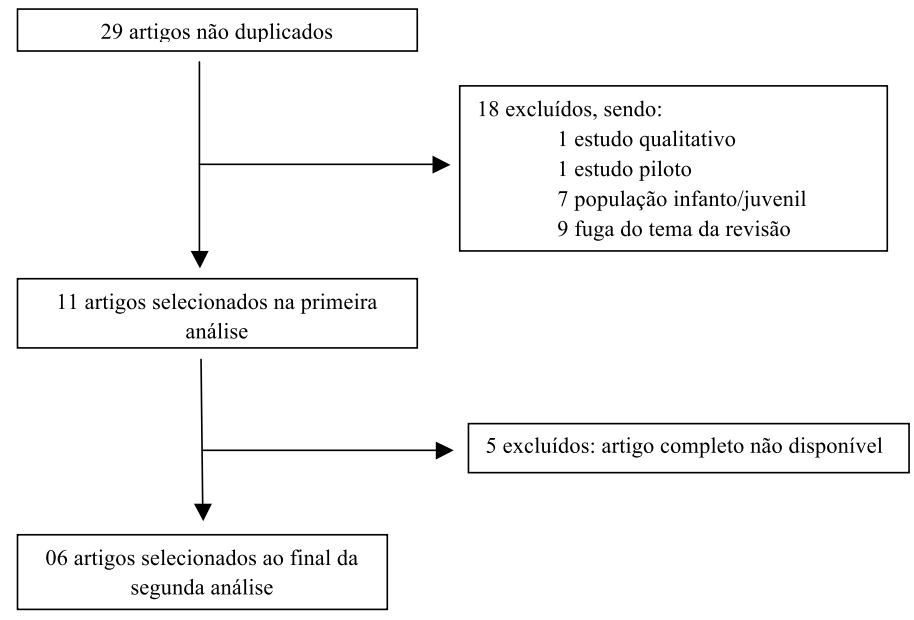

Figura 1 - Fluxograma das etapas da seleção dos artigos de interesse. 


\section{RESULTADOS E DISCUSSÃO}

Os estudos foram realizados na América Latina sendo a maioria deles no Brasi $\left.\right|^{9,10,12,14}$ e apenas um realizado no México. ${ }^{11}$ Os seis estudos (Tabela 1) avaliaram o estado nutricional através do IMC, sendo que dois deles avaliaram também a prevalência de sobrepeso em PV/HIV..$^{11,13}$ Outros avaliaram também a qualidade da dieta, ${ }^{14}$ estado nutricional, ${ }^{10,12}$ diagnóstico e características demográficas, ${ }^{11}$ riscos cardiovasculare ${ }^{12}$ e autorrelato de lipodistrofia. ${ }^{9}$

Todos os estudos avaliaram participantes com mais de 18 anos, HIV+ e em uso de TARV. Apenas um estudo comparou grupo HIV + com uso de TARV, com grupo HIV+ sem uso de TARV. ${ }^{9}$

Na Tabela 2 é possível observar a prevalência de excesso de peso, definida como somatório do sobrepeso e obesidade, verificando que variou entre $25 \%$ até $48,3 \%$. Estes dados já trazem a prevalência próxima dos estudos para população geral, como por exemplo, no Brasil, os dados populacionais descritos pelo Vigitel (Vigilância de fatores de risco e proteção para doenças crônicas por inquérito telefônico) em 2014 descrevem prevalência de excesso de peso de $52,5 \% .^{16}$

Estudo realizado por Soares et al. ${ }^{9}$ avaliou 227 participantes divididos em três grupos: grupo $1=92$ $\mathrm{HIV}+$ em uso de TARV e com autorrelato de lipodistrofia. Grupo 2 = 70 HIV+ em uso de TARV sem autorrelato de lipodistrofia, e grupo $3=65 \mathrm{HIV}+$ sem uso de TARV. O objetivo foi avaliar a prevalência de autorrelato de lipodistrofia e estado nutricional, e como ferramenta para alcançar os objetivos foram realizadas medidas antropométricas, cálculo de IMC, além da coleta de dados sociodemográficos e cálculo para risco de doenças cardiovasculares. O estudo de população predominantemente masculina (152 homens e 75 mulheres) identificou autorrelato de síndrome lipodistrófica por 33\% das mulheres e $59 \%$ entre os homens, além de $57,7 \%$ de risco de doença cardiovascular e 47,6\% de excesso de peso. ${ }^{9}$ Entretanto, foram utilizados peso e altura relatados pelos pacientes, o que pode ter subestimado os resultados.

Outros dois estudos que objetivaram avaliar estado nutricional de PV/HIV ${ }^{10,12}$ apontaram prevalência de excesso de peso em $37,1 \%$ e $42,6 \%$ dos participantes, respectivamente. Os dois também apontaram para maior taxa de excesso de peso em mulheres, sendo que em um deles a diferença chegou a ser até 3 vezes maior do que em homens. ${ }^{10}$ Além disso idade $<40$ anos, hipertensão, baixo nível educacional e tabagismo estavam relacionados ao excesso de peso dos participantes da pesquisa. ${ }^{10}$

A pesquisa realizada por Kroll et al., ${ }^{12}$ além de apontar para maior frequência de excesso de peso em mulheres, também concluiu que o risco cardiovascular era maior nesta população. Entretanto neste estudo não foi encontrado associação entre excesso de peso com o uso regular de TARV. ${ }^{12}$

A prevalência de $25 \%$ de excesso de peso foi relatada no estudo de Duran et al. ${ }^{14} \mathrm{O}$ estudo de corte transversal teve como objetivo descobrir a qualidade da dieta de PV/HIV, e concluiu que brasileiros que vivem com o vírus estão ficando com excesso de peso devido à má alimentação, onde $64,3 \%$ dos indivíduos apresentaram uma dieta inadequada. ${ }^{14}$

As pesquisas de Núñez-Rocha et. al e Mariz et al. ${ }^{11,13}$ tiveram como objetivo avaliar e estimar a preva-

Tabela 1 - Conteúdos online (webpage/blogs) com temas dedicados a promoção da saúde.

\begin{tabular}{|c|c|c|c|c|}
\hline Autor e Ano & Revista & País & Objetivo & Tipo de Estudo \\
\hline $\begin{array}{l}\text { Soares et al. }{ }^{9} \\
2015\end{array}$ & Revista Instituto Medicina Tropical & Brasil & $\begin{array}{l}\text { Avaliar a prevalência de autorrelato de lipo- } \\
\text { distrodia e estado nutricional de PV/HIV. }\end{array}$ & Corte Transversal \\
\hline $\begin{array}{l}\text { Senna et al. }{ }^{10} \\
2014\end{array}$ & Journal Health Population Nutrition & Brasil & Avaliar o estado nutricional de PV/HIV. & Corte Transversal \\
\hline $\begin{array}{l}\text { Núñez-Rocha et al. }{ }^{11} \\
2013\end{array}$ & Revista de Investigación Clínica & México & $\begin{array}{l}\text { Avaliar a associação entre o diagnostico, } \\
\text { características demográficas e o índice de } \\
\text { massa corporal, e prevalência de sobrepeso/ } \\
\text { obesidade em PV/HIV. }\end{array}$ & Corte Transversal \\
\hline $\begin{array}{l}\text { Kroll et al. }{ }^{12} \\
2012\end{array}$ & $\begin{array}{l}\text { Arquivos Brasileiros de Endocrino- } \\
\text { logia e Metabologia }\end{array}$ & Brasil & Descobrir o estado nutricional de PV/HIV & Corte Transversal \\
\hline $\begin{array}{l}\text { Mariz et al. }{ }^{13} \\
2011\end{array}$ & Caderno de Saúde Pública & Brasil & $\begin{array}{l}\text { Estimar a prevalência de magreza, sobrepeso/ } \\
\text { obesidade em PV/HIV e fatores associados. }\end{array}$ & Corte Transversal \\
\hline $\begin{array}{l}\text { Duran et al. }{ }^{14} \\
2008\end{array}$ & $\begin{array}{l}\text { Journal of Human Nutrition and } \\
\text { Dietetics }\end{array}$ & Brasil & Descobrir a qualidade da dieta de PV/HIV & Corte Transversal \\
\hline
\end{tabular}

Tabela 2 - Estudos incluídos: autor, número de pessoas avaliadas, características da população e prevalência de sobrepeso, obesidade e excesso de peso.

\begin{tabular}{|c|c|c|c|c|c|}
\hline Autor e Ano & $\mathbf{N}$ & População & Sobrepeso & Obesidade & Excesso de peso \\
\hline Soares et al. ${ }^{9}$ & 227 & $\begin{array}{c}\text { HIV + TARV + LD + }=92 \\
\text { HIV + TARV + LD- }=70 \\
\text { HIV + TARV - LD- }=65\end{array}$ & & & $47,6 \%$ \\
\hline Senna et al. ${ }^{10}$ & 235 & HIV + TARV + & $26 \%$ & $11,1 \%$ & $37,1 \%$ \\
\hline Núñez-Rocha et al. ${ }^{11}$ & 241 & HIV + TARV + & $35,8 \%$ & $12,5 \%$ & $48,3 \%$ \\
\hline Kroll et al. ${ }^{12}$ & 345 & HIV + TARV + & $34,2 \%$ & $8,4 \%$ & $42,6 \%$ \\
\hline Mariz et al. ${ }^{13}$ & 2.018 & HIV + TARV + & & & $32,1 \%$ \\
\hline Duran et al..$^{14}$ & 56 & HIV + TARV + & $25 \%$ & & $25 \%$ \\
\hline
\end{tabular}

Abreviações: HIV (virus da imunodeficiência humana); TARV (terapia antirretroviral); LD (lipodistrofia) 
lência de excesso de peso em PV/HIV e identificaram as taxas de $48,3 \%$ e $32,1 \%$, respectivamente. O primeiro constituiu população predominantemente masculina $(87 \%)$ e jovem (idade inferior a 50 anos). A prevalência de sobrepeso e obesidade foi de $35,8 \%$ e $12,5 \%$, respectivamente, apontando prevalência de excesso de peso de quase $50 \%$. Outro ponto relevante apontado pela pesquisa foi que os pacientes com maior IMC também tinham maior tempo de diagnóstico $(5,0 \pm 9,4$ anos). A hipótese dos autores é de que o aumento de peso é uma consequência da correta utilização da TARV, que promove um controle da progressão da doença. ${ }^{11}$

Ainda segundo estudo de Mariz et al., 61,8\% da população masculina e $54,6 \%$ da feminina eram eutróficos, e a taxa de obesidade até três vezes maior do que a de magreza, em ambas populações. ${ }^{13}$

O excesso de peso foi citado e discutido em todos os artigos, e a prevalência variou entre $25 \%$ a $48,3 \%$. Segundo os estudos descritos no presente trabalho, a prevalência de excesso de peso pode estar associada à idade, diabetes, ${ }^{13}$ a baixa qualidade da dieta, ${ }^{14}$ hipertensão, tabagismo, baixo nível educacional ${ }^{10} \mathrm{e}$ maior tempo de diagnóstico. ${ }^{11}$

\section{CONSIDERACִÕES FINAIS}

Apenas 06 artigos estavam disponíveis de acordo com os objetivos da presente revisão sistemática, o que demonstra ainda poucos estudos com este objetivo. Respondendo ao objetivo, a análise dos artigos selecionados nesta revisão apontou variação $25 \%$ a $48,3 \%$ de prevalência de excesso de peso em PV/HIV.

Conclui-se que o excesso de peso em pessoas que vivem com HIV vem sendo cada vez mais relatado em estudos abrangendo esta população. Estes estudos também apontam diversos fatores que podem estar associados ao excesso de peso, o que sugere a necessidade de um olhar mais abrangente para as novas adversidades enfrentadas por PV/HIV, fazendo-se necessário o desenvolvimento de estratégias que visem seu estado nutricional e consequentemente seu bem-estar e qualidade de vida.

\section{REFERÊNCIAS}

1. Alencar TMD, Nemes MIB. Velloso MA. Transformações da "aids aguda" para a "aids crônica": percepção corporal e intervenções cirúrgicas entre pessoas vivendo com HIV e aids: Cienc Saúde Coletiva 2008;13(6):1841-9. doi: 10.1590/ S1413-81232008000600019.

2. Segatto AFM, Freitas Junior IF, Santos VR, AlvesKCP, Barbosa DA, Portelinha Filho AM, Monteiro HL. Lipodystrophy in HIV/AIDS patients with different levels of physical activity while on antiretroviral therapy: Rev Soc Bras Med Trop 2011;44(4):420-4. doi: 10.1590/S003786822011000400004.

3. Tsuda LC, Silva MM, Machado AA, Fernandes AP. Body changes: antiretroviral therapy and lipodystrophy syndrome in people living with HIV/AIDS. Rev Latino-Am
Enfermagem 2012;20(5):847-53. doi: 10.1590/S010411692012000500005

4. Jaime PC, Florindo AA, Latorre MRDO, Segurado AAC. Central obesity and dietary intake in HIV/AIDS patients. Rev Saúde Pública 2006;40(4):634-40. doi: 10.1590/S003489102006000500012.

5. Jaime PC, Florindo AA, Latorre MRDO, Brasil BG, Santos ECM, Segurado AAC. Prevalência de sobrepeso e obesidade abdominal em indivíduos portadores de HIV/ AIDS, em uso de terapia anti-retroviral de alta potência. Rev Bras Epidemiol 2004;7(1):65-72. doi: 10.1590/S1415790X2004000100008.

6. Quaioti TCB, Almeida SS. Determinantes psicobiológicos do comportamento alimentar: uma ênfase em fatores ambientais que contribuem para a obesidade. Psicol USP 2006;17(4):193211. doi: 10.1590/S0103-65642006000400011.

7. Crum-Cianflone N, Tejidor R, Medina S, Barahona I, Ganesan A. Obesity among patients with HIV: the latest epidemic. AIDS Patient Care and STDS 2008;22(12):925-30. doi: 10.1089/apc.2008.0082.

8. Falco M, Castro ACO, Silveira EA. Nutritional therapy in metabolic changes in individuals with HIV/AIDS. Rev Saúde Pública 2012;46(4):737-46. doi: 10.1590/S003489102012005000050.

9. Soares LR, Silva DC, Gonsalez CR, Batista FG, Fonseca LA, Duarte AJ, Casseb J. Discordance between body mass index and anthropometric measurements among hiv-1-infected patients on antiretroviral therapy and with lipoatrophy/lipohypertrophy syndrome. Rev Inst Med Trop São Paulo 2015;57(2):105-10. doi: 10.1590/S003646652015000200002.

10. Senna AF, Oliveira SA, Velarde LG, Setubal S. Nutritional Status of HIV-positive Patients in Niteroi, Rio de Janeiro, Brazil. J Health Popul Nutr 2014;32(4):595-9.

11. Núñez-Rocha GM, Wall KM, Chávez-Peralta $M$, SalinasMartínez AM, Benavides-Torres RA. Nutritional care, time period since diagnosis, demographics and body mass index in HIV/AIDS patients. Rev Invest Clin 2013;65(4):291-9.

12. Kroll AF, Sprinz E, Leal SC, Labrêa MG, Setubal S. Prevalence of obesity and cardiovascular risk in patients with HIVIAIDS in Porto Alegre, Brazil. Arq Bras Endocrinol Metab 2012;56(2):13741. doi: $10.1590 /$ S0004-27302012000200007.

13. Mariz CA, Albuquerque MFPM, Ximenes RAA, Melo HRL, Bandeira F, Braga e Oliveira TG, Carvalho EH, Silva AP, Miranda Filho DB. Body mass index in individuals with HIV infection and factors associated with thinness and overweight/ obesity: Cadernos Saúde Pública 2011;27(10):1997-2008. doi: 10.1590/S0102-311X2011001000013.

14. Duran AC, Almeida LB, Segurado AA, Jaime PC. Diet quality of persons living with HIV/AIDS on highly active antiretroviral therapy. J Hum Nutr Diet 2008;21(4):346-50. doi: 10.1111/j.1365-277X.2008.00886.x

15. Castrighini CC, Reis RK, Neves LAS, Brunini S, Canini SRMS, Gir E. Avaliação da autoestima em pessoas vivendo com HIV/AIDS no município de Ribeirão Preto-SP: Texto Contexto - Enferm 2013;22(4):1049-55. doi: 10.1590/ S0104-07072013000400022.

16. Brasil. Minitério da Saúde. Vigilância de fatores de risco e proteção para doenças crônicas por inquérito telefônico (VIGITEL). Brasília, 2014.z

Como citar: PINHAL, Fernanda Cristina Gomes. Prevalência de excesso de peso em pessoas que vivem com HIV: uma revisão sistemática. Cinergis, Santa Cruz do Sul, v. 17, n. 4, out. 2016. ISSN 2177-4005. Disponível em: <https://online.unisc.br/ seer/index.php/cinergis/article/view/8141 >. Acesso em: 11 out. 2016. doi:http://dx.doi.org/10.17058/cinergis.v17i4.8141. 